\title{
Darmmikrobiom bei primären Hirntumoren
}

\author{
Datenlage und Hypothesen
}

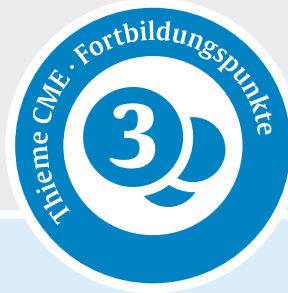

Tareq M. Anssar, Peter Hau

Wilhelm Sander-Therapieeinheit NeuroOnkologie und Klinik und Poliklinik für Neurologie, Universität Regensburg

\section{ZUSAMMENFASSUNG}

Primäre Hirntumoren kommen weniger häufig vor als andere Tumorentitäten. Das Glioblastom ist der häufigste primäre Hirntumor bei Erwachsenen und hat eine sehr schlechte Prognose. Die Pathophysiologie vieler Hirntumoren ist nur unzureichend verstanden. Eine kurative Therapie existiert bis auf wenige Ausnahmen nicht. Bei einigen soliden und hämatologischen Tumoren konnte in den letzten Jahren ein Zusammenhang zwischen Tumorentstehung und -progression mit
Veränderungen im intestinalen Mikrobiom festgestellt werden. Zusammenhänge zwischen Mikrobiota und primären Hirntumoren wurden hingegen nicht publiziert.

In der vorliegenden Arbeit werden Mechanismen dargestellt, die bei einer möglichen Interaktion zwischen Mikrobiom und Hirntumoren eine Rolle spielen können. Dabei steht die Schranke vom Darmepithel zum peripheren Blut und vom peripheren Blut zum Gehirn im Mittelpunkt des Interesses. Im Hinblick auf die Darm-Blutschranke sind Interaktionen zwischen Mikrobiom und Immunphänotyp sowie Metaboliten im peripheren Blut bekannt. Im Hinblick auf die Blut-Hirnschranke bestehen mögliche Assoziationen des Immunphänotyps und der Metaboliten im peripheren Blut mit der Immuninfiltration im Gehirn und der Induktion pathogeneserelevanter Signalkaskaden.

\section{Inzidenz, Therapie und Prognose der primären Hirntumoren}

Primäre Hirntumoren sind im Vergleich zu anderen Tumorentitäten eher selten. Glioblastome sind die häufigsten hirneigenen Tumoren mit einer Inzidenz von etwa 3,2/100 000 Einwohnern pro Jahr [1]. Bei einem Großteil der Glioblastome handelt es sich um primäre Formen, während sich etwa $10 \%$ sekundär aus niedrig malignen Gliomen entwickeln [2]. Trotz aktueller Standardtherapie, bestehend aus Resektion, adjuvanter kombinierter Radiochemotherapie, adjuvanter Chemotherapie mit Temozolomid und begleitender Anwendung von Tumortherapiefeldern liegt das mediane Überleben beim Glioblastom bei etwa 20,9 Monaten [3]. Bei anderen primären Hirntumoren liegen die Überlebenszeiten abhängig von der Tumorgenetik zwischen den Überlebenszeiten beim Glioblastom und nahezu uneingeschränkter Lebenserwartung.

\section{Hinweise auf Interaktionen zwischen Mikrobiom und ZNS}

Mikrobiom und zentrales Nervensystem (ZNS) stellen 2 Systeme dar, die sich direkt oder indirekt bidirektional beeinflussen können. Zahlreiche Interaktionen zwischen diesen beiden Komponenten legen einen aktiven Austausch und damit auch eine Relevanz bei Hirntumoren nahe. Auf diese Aspekte gehen zahlreiche Artikel in diesem Themenheft ein. Der folgende Absatz gibt daher nur einen einführenden Kurzüberblick über diese Interaktionen.
Das Mikrobiom spielt in der frühen Kindesentwicklung, der normalen Entwicklung und im Alter eine bedeutende Rolle [4]. Mehrere Studien haben insbesondere im Tiermodell den Einfluss des Mikrobioms auf die Kognition und auf eine demenzielle Entwicklung gezeigt [5]. Es konnte zudem gezeigt werden, dass das Mikrobiom eine Rolle bei der Gedächtnisfunktion [6], beim emotionalen Lernen und der emotional konnotierten Entscheidungsfindung [7] und bei weiteren Aspekten der Kognition hat [8]. Zudem spielt das Mikrobiom bei Angststörungen und Depressionen eine Rolle [9]. In einer Kohorte mit 1054 Probanden konnte ein Zusammenhang zwischen den Mikrobiota und der Lebensqualität nachgewiesen werden [10].

Auch wenn die Mechanismen nicht in jedem Fall vollständig aufgeklärt sind, belegen die zahlreichen Interaktionen doch eindeutig den Zusammenhang zwischen Mikrobiota und dem gesunden und erkrankten Gehirn. Mögliche Mechanismen dieser Interaktion zwischen Mikrobiota und ZNS beinhalten eine Aktivierung des N. vagus [11], neuroimmunologische Signalwege [12], mikrobielle Metaboliten [13] und mikrobiotaabhängige Neurotransmitter wie das glutaminerge System [14].

\section{Lokale Veränderungen am Darm und Tumorentstehung}

Mit Etablierung moderner Sequenzierungsmethoden konnte bei mehreren soliden Tumoren ein Zusammenhang zwischen mikrobiellen Spezies und der Tumorentwicklung festgestellt werden. In dieser Hinsicht besonders gut un- 
tersucht ist das kolorektale Karzinom (CRC) [15]. In mehreren Studien bei Patienten mit CRC konnte gehäuft Fusobacterium nucleatum im intestinalen Mikrobiom nachgewiesen werden [16, 17]. Diese Akkumulation führt über direkte Invasion des Bakteriums in das Darmepithel und Cyclooxygenase-2 (Cox-2), Interleukin (IL)-6 und Tumornekrosefaktor (TNF)- $\alpha$-vermittelte Inflammation zu einer deutlich höheren Inzidenz des CRC [17]. Allerdings ist vermutlich nicht Fusobacterium nucleatum alleine, sondern die Zusammensetzung der gesamten Mikrobiota im Darm verantwortlich. Auch bei anderen Entitäten wie dem Ösophagus- [18] , dem hepatozellulären (HCC) [19] oder dem Mammakarzinom [20] gibt es Hinweise auf solche Zusammenhänge.

Eine Störung der Barrierefunktion oder eine Verschiebung des Gleichgewichts zwischen regulatorischen T-Zellen (Tregs) und Typ 17 T-Helferzellen (Th17)-Zellen zugunsten der Tregs im Darm, erlaubt Bakterienbestandteilen wie Lipopolysaccharid (LPS) zum Epithel zu gelangen [21]. Diese Translokation von Bakterienbestandteilen scheint bei der Entstehung des HCC ebenfalls eine entscheidende Rolle zu spielen [19]. Ein Erklärungsansatz ist, dass bestimmte Bestandteile der Bakterien wie LPS über das Blut in die Leber gelangen und dort durch Aktivierung von pattern recognition Rezeptoren (PRR) wie Toll-like-Rezeptoren (TLRs) oder NOD-like Rezeptoren (NLRs) und konsekutiv die Freisetzung proinflammatorischer Zytokine zunächst eine lokale Entzündungsreaktion unterhalten und längerfristig die Progression des HCC fördern [19]. Auch durch die Mikrobiota entstehende Metabolite können lokal an der Tumorentstehung beteiligt sein [22].

Schließlich konnte für einige Bakterienspezies eine sekundäre Aktivierung von pathogeneserelevanten Signalwegen nachgewiesen werden. So führt das von Bacteroides fragilis gebildete Toxin zunächst zu einer Spaltung von E-Cadherin und sekundär zu einer Aktivierung des $\beta$-Catenin/Wnt-Signalwegs und somit zu einer vermehrten c-Myc-Expression [23]. Außerdem kommt es im Kolon zu einer nuclear factor ,kappa-light-chain-enhancer' of activated B-cells (NF-kB)- und signal transducer and activator of transcription 3 (STAT3)-Aktivierung [24]. Auch durch Fusobacterium nucleatum wird die kolorektale Carcinogenese über den $\beta$-Catenin/Wnt-Signalweg und NF-кB-Aktivierung gefördert [17, 25]. Beide Signalwege nehmen entscheidenden Einfluss auf die Tumorprogression.

\section{Schrankenfunktion zwischen Mikrobiota und primärem Hirntumor}

Zur Rolle der Mikrobiota bei primären Hirntumoren liegen keine Publikationen vor. Insbesondere wurde der Einfluss eines möglicherweise veränderten Mikrobioms auf den peripheren Immunphänotyp oder das Metabolom und letztlich auf den Tumor im ZNS an beiden Schranken nicht untersucht.
Der Gastrointestinaltrakt enthält sowohl eine riesige $\left(3,8 \times 10^{13}\right)$ und sehr diverse Population an Mikrobiota als auch eine üppige Population an Tregs und Th17-Zellen [26]. Wichtig für die Interaktion zwischen Mikrobiota und nicht im Darm angesiedelten soliden Tumoren ist die Integrität der Barriere zwischen Darm und peripherem Blut. Bei Gesunden besteht über eine weitestgehend bakterienfreie Mukusschicht eine anatomische Abgrenzung von Mikrobiom und Epithelzellen des Wirts [27]. Das ZNS galt aufgrund der Blut-Hirnschranke und der damit verbundenen geringen Zahl antigenpräsentierender Zellen und T-Zellen lange als immunprivilegiertes Organ, wenngleich in den vergangenen Jahren zunehmend Zweifel an diesem Konzept aufkamen [28]. Bei Krankheiten wie Autoimmun-Enzephalitiden [29] und bei ZNS-Tumoren [30] ist zudem seit längerem bekannt, dass es aufgrund einer gestörten Barrierefunktion zur Migration von Immunzellen aus der Peripherie in das ZNS kommt. Interaktionen zwischen Mikrobiom und primärem Hirntumor sind also bei Veränderungen an beiden Schranken durchaus denkbar. Die Blut-Hirnschranke ist zumindest bei höher malignen primären Hirntumoren immer gestört.

\section{Mechanismen der Beeinflussung distanter Tumoren durch das Mikrobiom}

\section{Mikrobiom und Immunsystem}

Die erste Achse einer möglichen Interaktion zwischen Mikrobiota und entfernt lokalisierten soliden Tumoren ist die Veränderung der Immunzellpopulation im peripheren Blut durch veränderte Mikrobiota. Das mikrobielle Wachstum wird durch eine Vielzahl an Immunzellen im gut-associated lymphoid tissue (GALT), durch mukosaassoziierte Th17-Zellen sowie durch die Produktion von Immunglobulin A (IgA) kontrolliert [21]. Umgekehrt konnte gezeigt werden, dass auch Mikroorganismen die Entwicklung, Stabilität und Funktion von ortsständigen Tregs regulieren können [31, 32]. Diese sind nach Transition in das periphere Blut effektive Regulatoren der Immunantwort [33].

Th17-Lymphozyten sind IL-17-sezernierende T-Helferzellen und werden wie Tregs durch die Mikrobiota induziert [34]. Die Expansion von Th17-Zellen kann durch Mikrobiota aber auch gehemmt werden. So kommt es beispielsweise durch Bindung von aus Bakterien wie Bacteroides fragilis stammendem Polysaccharid A (PSA) an TLR2-zu einer Zunahme der IL-10-Produktion, welche die Th17-Expansion hemmt [35]. Andererseits führt eine Kolonisation mit PSA-produzierenden Bacteroides fragilis zu einer Erhöhung der Zahl systemischer cluster of differentiation 4 (CD4)-positiver T-Zellen [36]. Durch diese Regulationen können auch Effekte an weit entfernten Organen induziert werden. So kann zum Beispiel eine durch Mikrobiota induzierte Th17-Vermehrung im Tiermodell zu extraintestinalen Erkrankungen wie einer autoimmunen Enzephali- 


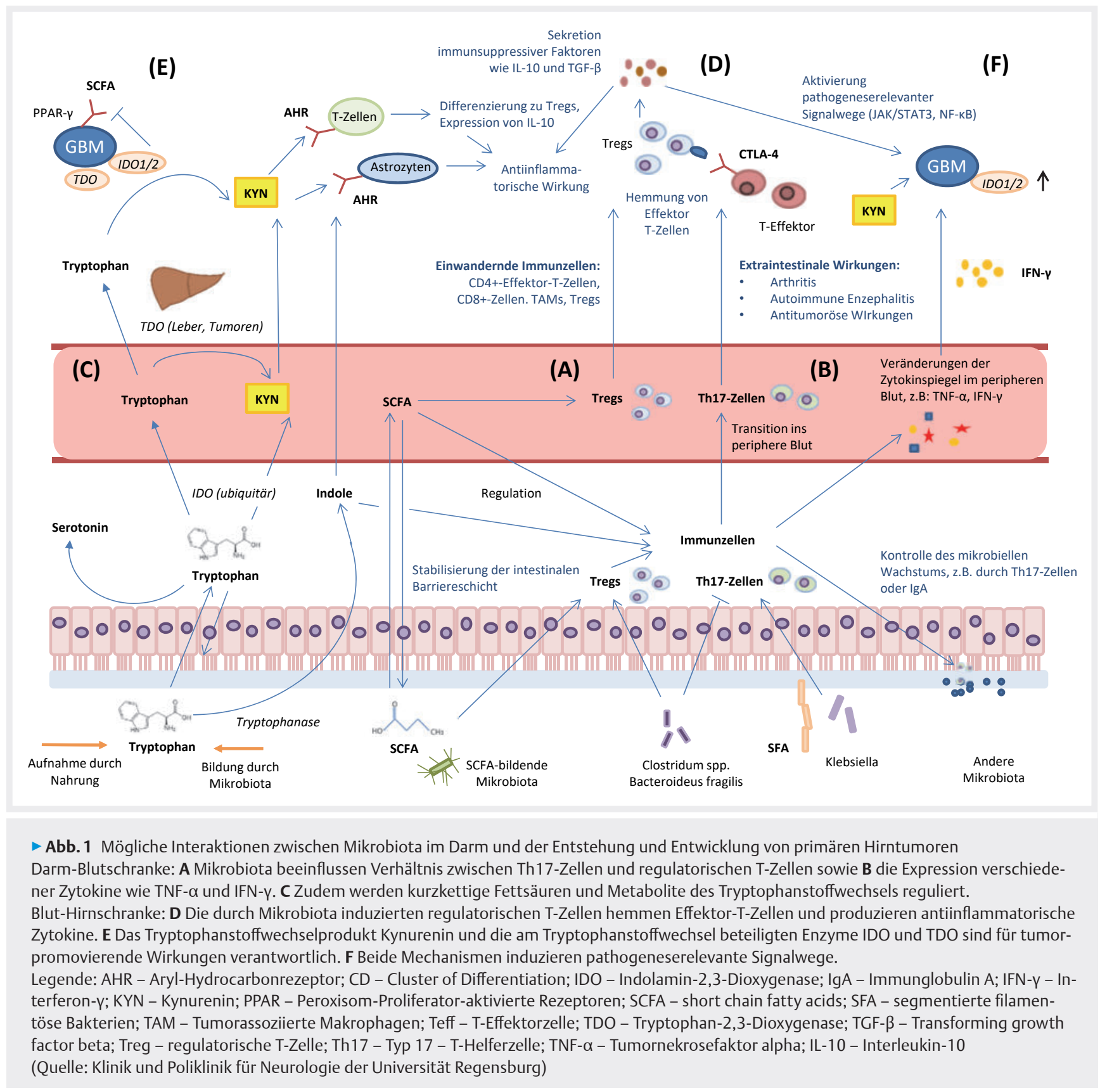

tis [12] führen. Auch therapeutische Interventionen haben Auswirkungen auf das Immunsystem. Beispielsweise können Chemotherapeutika die Einwanderung von Th17 in den Tumor und damit die Immunreaktion gegen solide Tumoren beeinflussen [37].

Neben diesen zellulären Veränderungen im peripheren Blut kommt es mikrobiotabedingt auch zu Veränderungen von Zytokinkonzentrationen. Beispielsweise sind die Konzentrationen von TNF- $\alpha$ und Interferon (IFN)-y stark abhängig von Veränderungen im Mikrobiom. Andere Zytokine wie IL-1 $\beta$, IL-6, IL-17 und IL-22 sind weniger stark, jedoch oft spezifischer mit bestimmten Mikrobiota assoziiert [38]. Zur Beeinflussung primärer Hirntumoren durch Mikrobiota über das Immunsystem existieren nur indirek- te Daten. Das lokale Immunzellinfiltrat spielt jedoch eine wichtige Rolle in der Pathogenese von Gliomen. An diesem Immunzellinfiltrat sind CD4+-Effektor-Zellen, CD8 ${ }^{+}$-Zellen, Treg-Zellen, tumorassozierte Makrophagen (TAMs) und aus dem Knochenmark stammenden Suppressorzellen (MDSCs) beteiligt [39-41]. T-Zellen machen etwa 0,25\% [42] und TAMs bis zu 12\% [43] der Zellen im Tumorgewebe aus. Die Prognose von Patienten mit Glioblastom korreliert dabei mit der endogenen T-Zell-Antwort gegen den Tumor. Insbesondere die lokale Akkumulation und Aktivierung von Effektor-T-Zellen und zytotoxischen T-Zellen im Tumorgewebe und die Rekrutierung und Aktivierung von Makrophagen im Tumor spielen hierbei eine bedeutende Rolle [41, 44, 45]. 
Die Komposition des lokalen Immuninfiltrats im primären Hirntumor könnte durchaus durch Mikrobiota indirekt beeinflusst werden. Mikrobiota induzieren ortsständige Tregs [31, 32], die nach Transition in das periphere Blut die systemische Immunantwort effektiv regulieren [33]. Bei Patienten mit primären Hirntumoren nimmt das Verhältnis von Tregs im peripheren Blut proportional zum Gesamtpool der CD4-positiven Zellen zu [40]. Die genaue Ursache dieser relativen Zunahme ist nicht geklärt. Im Serum von Patienten mit Glioblastom sind zudem starke Veränderungen der Zytokinexpression nachweisbar [46, 47]. In der Folge hemmen lokale immunsuppressive Faktoren wie IL-10 und transforming growth factor (TGF)- $\beta[48,49]$ sowie die Expression von Immun-Checkpunkten wie Cytotoxic T lymphocyte associated molecule (CTLA)-4 [50] durch Tregs antigenspezifische T-Zell-Antworten. Eine lokale Sekretion bestimmter Substanzen wie chemokine ligand 22 (CCL22) durch Glioblastomzellen scheint zudem die Rekrutierung von Tregs in das Mikromilieu des Tumors zu begünstigen [51]. Auch eine vermehrte Expression bestimmter Metabolite bzw. kinetikbestimmender Enzyme des Metaboloms wie Indoleamin-2,3-Dioxygenase (IDO) durch Tumorzellen führt zu einer vermehrten Rekrutierung von Tregs im Tumorgewebe und ist mit einer schlechteren Prognose assoziiert [52]. Eine Depletion der Tregs führt zu einer Normalisierung der T-Zell-Funktionen [40]. Andererseits konnten andere Studien keine signifikante Assoziation zwischen vermehrter Infiltration durch Tregs und einer schlechteren Prognose feststellen [53].

Entscheidend für das Schicksal des Tumors sind letztlich pathogeneserelevante tumorpromovierende oder -inhibierende Signalkaskaden im Tumor und dessen Mikroumgebung. Lokal im ZNS immunzellsezernierte Zytokine wie Fas-Ligand, TNF- $\alpha$, IFN- $\gamma[54]$ oder TGF- $\beta[55,56]$ können neben ihren immunmodulierenden Effekten verschiedene für den Glioblastom-Phänotyp relevante Zytokine wie IL-6 und IL-8 [57] und Signalwege wie Januskinase (JAK)/ STAT-3 sowie Transkriptionsfaktoren wie NF-kB [57] induzieren. Diese spielen in der Pathophysiologie primärere Hirntumoren eine entscheidende Rolle.

\section{Mikrobiom und Tumormetabolismus}

Die zweite Achse einer möglichen Interaktion zwischen Mikrobiota und entfernt lokalisierten soliden Tumoren ist eine Veränderung des Metaboloms mit sekundären Effekten auf solide Tumoren. Inzwischen ist in Tiermodellen und teilweise auch im Menschen gut belegt, dass das intestinale Metabolom direkten Einfluss auf des Metabolom im peripheren Blut und somit möglicherweise auch das Metabolom im Parenchym distanter Tumoren hat [58]. Der Tumormetabolismus spielt eine entscheidende Rolle in der Entstehung und Aufrechterhaltung von Hirntumoren [59].

Der Fettstoffwechsel und dabei entstehende Metabolite, beispielsweise kurzkettige Fettsäuren (engl. short chain fatty acids, SCFA) wie Butyrat, sind abhängig von der Zusammensetzung der intestinalen Mikrobiota und bedeutend für die Aufrechterhaltung der intestinalen Barrierefunktion und Produktion antimikrobieller Peptide im Darm [60]. SCFA können problemlos vom Darm in das Blut gelangen und damit beispielsweise die Differenzierung von regulatorischen T-Zellen nicht nur im Kolon, sondern auch in der Peripherie fördern [32, 61]. Zudem haben SCFAs einen Einfluss auf die Reifung und Erhaltung der Mikroglia im Gehirn [13]. Hier wird ein direkter Zusammenhang zwischen dem intestinalen Mikrobiom, dem systemischen Metabolom und der systemischen Immunantwort im peripheren Blut sichtbar.

Ein weiterer noch besser untersuchter Mechanismus ist abhängig von Tryptophan, einer essenziellen proteinogenen Aminosäure, die zusammen mit ihren Metaboliten eine Schlüsselrolle in der Koordination von Nährstoffangebot und Stoffwechselvorgängen spielt [62]. Tryptophan wird im Dünndarm aufgenommen und kann dann von Darmbakterien direkt zu einer Reihe von Indolderivaten katabolisiert werden, die eine Schlüsselrolle in der Aufrechterhaltung der Mikrobiota-Balance im Dickdarm spielen [63]. Darüber hinaus wird Tryptophan für die Serotoninsynthese verwendet [64] und in den Kynurenin-Stoffwechselweg eingeschleust [65]. Die Verfügbarkeit von Tryptophan ist dabei abhängig von der mikrobiellen Flora. Intestinale Mikrobiota können abhängig vom jeweiligen Bakterium zu einer Erhöhung [66] oder zu einer Reduktion [67] der Kynureninkonzentration im Blut führen. Es konnte zudem gezeigt werden, dass eine Erhöhung der Kynureninspiegel im Blut mit einer Erhöhung der Kynurenin-, Chinolinsäure- und Kynurensäurespiegel im Liquor einhergehen [68].

Im Vergleich keimfreier Mäuse mit Mäusen mit vorhandenen Mikrobiota unterscheidet sich das Metabolom zwischen beiden Gruppen, insbesondere in Bezug auf Aminosäuren aus dem Tryptophanstoffwechsel, fundamental [69]. Bei keimfreien Mäusen konnten dabei erhöhte Tryptophanspiegel im Plasma und erhöhte Spiegel der Metabolite von Tryptophan im Hippokampus gemessen werden $[65,69]$. Der größte Teil des verfügbaren Tryptophans wird in den Kynurenin-Stoffwechselweg eingeschleust und unterliegt der Kontrolle durch die geschwindigkeitsbestimmenden Enzyme IDO1, IDO2 und tryptophan-2,3-dioxygenase (TDO) [62]. Tryptophan und insbesondere seine beim Abbau entstehenden Katabolite spielen eine wichtige Rolle bei der Unterdrückung lokaler Immunantworten und bei der Malignisierung von Tumorzellen [70-72]. Tryptophan reguliert dabei insbesondere das Verhältnis zwischen Th17- und Treg-Zellen [73, 74]. Zahlreiche Studien haben hierbei gezeigt, dass IDO1 - teils sezerniert aus Tumorzellen - Tregs aktiviert oder differenziert und damit T-Zell-Antworten supprimiert [73, 74]. Die Proliferation von Effektor-T-Zellen und natürlichen Killerzellen (NK) wird durch IDO und Kynurenin gehemmt [75]. Bei Th1-Zellen 
kann durch Metabolite des Kynurenin-Stoffwechsels Apoptose induziert werden [76]. Auch TDO, das in humanen Tumorzellen Tryptophan zu Kynurenin umwandelt, wirkt über eine Inhibition der T-Zell-Proliferation zusätzlich immunsuppressiv [70, 71].

Bei diesen Vorgängen spielt der aryl hydrocarbon receptor (AHR) [77] eine entscheidende Rolle. Dabei handelt es sich um einen ligandenaktivierten Transkriptionsfaktor, der unter anderem auf Astrozyten exprimiert wird und die Immunantwort moduliert [78], sowie an der Differenzierung von Treg- und Th17-Zellen beteiligt ist [79]. Dabei kann AHR nicht nur durch vom Wirt produzierte endogene Metabolite des Tryptophanstoffwechsels wie Kynurenin aktiviert werden [70, 77], sondern auch durch Tryptophanmetabolite des katabolen Bakterienstoffwechsels wie mittel Tryptophanase entstehendes Indol [63], was eine Abhängigkeit von Mikrobiota nahelegt.

Für primäre Hirntumore ist die Datenlage deutlich schwächer. Es existieren aber auch hier indirekte Hinweise für einen Einfluss der Mikrobiota über den systemischen und lokalen Metabolismus auf das Schicksal des Tumors im ZNS. Bei Patienten mit Gliomen wurden reduzierte Tryptophanspiegel im Serum gefunden [80]. Zudem führt eine Aktivierung von IDO1 und TDO zu einer Akkumulation von Kynurenin und von Quinolonsäure und zur lokaler Immunsuppression [70, 71, 80, 81]. Interessanterweise wird die Expression von IDO durch immuninfiltrationskonnektierte Zytokine wie beispielsweise IFN-y verstärkt [82]. Die Expression von IDO in Hirntumoren ist dabei negativ mit dem Überleben assoziiert [52]. Neben den immunsuppressiven Wirkungen können Tryptophanentzug und mit dem Tryptophanstoffwechsel verbundene Enzyme wie TDO bei anderen Tumoren die Tumorzellinvasion und -proliferation verstärken sowie die Induktion von Apoptose verhindern [83]. Ähnliche Mechanismen sind auch beim Glioblastom zu erwarten.

\section{Mikrobiom und pathogeneserelevante Signalwege}

Die dritte Achse einer möglichen Interaktion zwischen Mikrobiota und entfernt lokalisierten soliden Tumoren ist die Aktivierung bestimmter Signalwege im Tumor durch Mikrobiota. Dieser Mechanismus tritt in der Regel als Folge von Ereignissen wie Verschiebungen in der lokalen Immunkontrolle oder Metabolitenausstattung auf. Die im Zusammenhang mit anderen soliden Tumoren genannten Signalwege spielen auch bei primären Hirntumoren eine bedeutsame Rolle. Allerdings werden die genannten Signalwege bei gastrointestinalen Tumoren durch eine direkte Interaktion der Mikrobiota mit dem Parenchym angestoßen $[15,17]$. Bei primären Hirntumoren ist dagegen eher eine indirekte Stimulation von Signalwegen über aus dem peripheren Blut in das ZNS invadierte Immunzellen oder Metabolite im Tumorparenchym zu erwarten.
Einige Kandidaten-Signalwege für solche Interaktionen sind gut untersucht. Ein Zusammenhang mit dem Mikrobiom wurde nicht hergestellt. Der kanonische (B-Catenin) und nicht kanonische WNT-Signalweg reguliert das Schicksal von Progenitorzellen des Glioblastoms und damit potenziell die Pathogenese dieser Tumoren [84]. STAT-3 bzw. der JAK/STAT-Signalweg ist ein Knotenpunkt der Tumorentwicklung beim Glioblastom [85] und wird durch aus Immunzellen sezernierte Zytokine wie IL-6 induziert [86]. Der Signalweg vermittelt Resistenz gegen Therapie, moduliert die Immunantwort gegen Glioblastome und fördert die Migration und Invasion von Glioblastom-Progenitorzellen im Parenchym $[87,88]$. NF-кB als ubiqitärer Transkriptionsfaktor mediiert den mesenchymalen Phänotyp im Glioblastom, der u. a. mit einer inflammatorischen Mikroumgebung, gesteigerter Invasion und Resistenz gegen programmierten Zelltod einhergeht [89]. NF-кB wird durch verschiedene Tyrosinkinasen aktiviert und reguliert zahlreiche Down-stream-Transkriptionsfaktoren wie z. B. STAT-3, CCAAT/enhancer-binding-protein (C/EB$P)$ - $ß$ und sezernierte Faktoren wie inflammatorische Zytound Chemokine (z. B. TNFo, TGF-ß, IL-8, IL-6) [89]. NF-kB und JAK/STAT-3 sind bei der Initiierung und Aufrechterhaltung des mesenchymalen Phänotyps eng verknüpft und agieren synergistisch, teils unter Aktivierung von immunmodulierenden Zytokinen [90]. Peroxisome Proliferator-Activated Rezeptoren (PPAR) sind Transkriptionsfaktoren, die durch physiologische Liganden wie beispielsweise Metabolite aus dem Aminosäure- und Fettstoffwechsel induziert werden können [91] und in der Signaltransduktion beim Glioblastom eine Rolle spielen [92]. Teils widersprüchliche In-vitro- und In-vivo-Ergebnisse legen je nach Kontext tumorpromovierende oder proliferations- und migrationshemmende, apoptoseinduzierende sowie immunmodulierende Effekte nahe [92]. Epidemiologische Studien weisen darauf hin dass PPAR- $\gamma$-Agonisten bei Patienten mit Glioblastom klinisch relevant sind [93]. ID01 inhibiert das Ansprechen auf PPAR-Agonisten [94]. Induzierte oder inhibierte Signalwege beeinflussen bei primären Hirntumoren auf vielschichtige Weise prognoserelevante Effekte wie Apoptose und Autophagie [95] und führen beispielsweise über NF-kB-, Zinc finger E-box-binding homeobox 1 (ZEB1)- oder STAT3-abhängige Mechanismen zu einer Transdifferenzierung von einem proneuralen hin zu einem mesenchymalen Phänotyp des Glioblastoms [96, 97]. Dieser Phänotyp zeichnet sich unter anderem durch eine erhöhte Invasivität der Tumorzellen aus, enthält vermehrt tumorinfiltrierende Lymphozyten und ist mit einer schlechteren Prognose assoziiert [96, 98]. Auch der $\beta$-Catenin/Wnt-Signalweg spielt eine Rolle bei der Entwicklung des mesenchymalen Phänotyps und einer erhöhten Invasivität bei Glioblastomen [84]. Hier bestehen also zahlreiche potenzielle Vernetzungen vom Metabolom zu bei Gliomen relevanten Signalwegen und Rezeptoren. 


\section{FAZIT FÜR DIE PRAXIS}

Interaktionen zwischen der Zusammensetzung der Mikrobiota im Darm und der Entstehung und Entwicklung von Hirntumoren sind wahrscheinlich. Mikrobiota können abhängig von der Integrität der Darm-Blutschranke die Barriere zwischen Darm und Blut überwinden und den peripheren Immunphänotyp - insbesondere das Verhältnis zwischen Th17-Zellen und Tregs - sowie die Expression verschiedener Zytokine wie TNF- $\alpha$ und IFN- $\gamma$ beeinflussen. Außerdem haben die Mikrobiota für die Regulation pathogeneserelevanter Stoffwechselprodukte wie kurzkettige Fettsäuren oder Metabolite des Tryptophanstoffwechsels eine fundamentale Bedeutung.

Diese mikrobiotainduzierten Mechanismen beeinflussen möglicher Weise auch primäre Hirntumoren in bedeutsamer Weise. Die durch verschiedene Mikrobiota induzierten Tregs spielen eine entscheidende Rolle für die Aufrechterhaltung des immunsuppressiven Mikromilieus beim Glioblastom. Auch das Tryptophanstoffwechselprodukt Kynurenin und die am Tryptophanstoffwechsel beteiligten Enzyme IDO und TDO könnten, z. B. über die Induktion oder Hemmung wichtiger Signalkaskaden, für tumorpromovierende Wirkungen verantwortlich sein. Aktuell sind weitere wissenschaftliche Untersuchungen nötig, um die bei primären Hirntumoren tatsächlich involvierten Mechanismen exakt zu identifizieren. Dabei werden sich mit einiger Wahrscheinlichkeit Angriffspunkte im Immunsystem oder an Schlüsselmechanismen des Tumormetabolismus ergeben, aus denen später neue und dringend benötigte Therapieoptionen entwickelt werden können.

\section{Interessenkonflikt}

Erklärung zu finanziellen Interessen

Forschungsförderung erhalten: nein; Honorar/geldwerten Vorteil für Referententätigkeit erhalten: ja; Bezahlter Berater/interner Schulungsreferent/Gehaltsempfänger: ja; Patent/Geschäftsanteile/Aktien (Autor/Partner, Ehepartner, Kinder) an Firma (Nicht-Sponsor der Veranstaltung): nein; Patent/Geschäftsanteile/Aktien (Autor/Partner, Ehepartner, Kinder) an Firma (Sponsor der Veranstaltung): nein.

Erklärung zu nicht finanziellen Interessen

Die Autoren geben an, dass kein Interessenkonflikt besteht. Mitglied im Steering Committee der Hirntumorgruppe der EORTC, assoziertes Mitglied im Vorstand der NeuroOnkologischen Arbeitsgemeinschaft (NOA), Mitglied der Kommission Neuroonkologie der Deutschen Gesellschaft für Neurologie.

\section{Danksagung}

Diese Arbeit wurde unterstützt durch die Else-KrönerFresenius-Stiftung.

\section{Korrespondenzadresse}

Prof. Dr. med. Peter Hau

Wilhelm Sander-Therapieeinheit NeuroOnkologie und Klinik und Poliklinik für Neurologie Universität Regensburg

Universitätsstr. 84, 93053 Regensburg

Tel. 0941/9418083, Fax 0941/94163013

peter.hau@ukr.de

\section{Literatur}

[1] Ostrom QT, Gittleman H, Truitt G, et al. CBTRUS Statistical Report: Primary Brain and Other Central Nervous System Tumors Diagnosed in the United States in 2011-2015. Neuro-Oncology 2018; 20: iv1-iv86. doi:10.1093/neuonc/ noy 131

[2] Ohgaki H, Kleihues P. The definition of primary and secondary glioblastoma. Clinical Cancer Research 2013; 19: 764-72. doi:10.1158/1078-0432.CCR-12-3002

[3] Stupp R, Taillibert S, Kanner A, et al. Effect of Tumor-Treating Fields Plus Maintenance Temozolomide vs Maintenance Temozolomide Alone on Survival in Patients With Glioblastoma: A Randomized Clinical Trial. JAMA 2017; 318: 2306-16. doi:10.1001/jama.2017.18718

[4] Zhuang L, Chen H, Zhang S, et al. Intestinal Microbiota in Early Life and Its Implications on Childhood Health. Genomics Proteomics Bioinformatics 2019; 17: 13-25. doi:10.1016/j.gpb.2018.10.002

[5] Sochocka M, Donskow-Lysoniewska K, Diniz BS, et al. The Gut Microbiome Alterations and Inflammation-Driven Pathogenesis of Alzheimer's Disease - a Critical Review. Mol Neurobiol 2019; 56: 1841-51. doi:10.1007/s12035-018$1188-4$

[6] Gareau MG, Wine E, Rodrigues DM, et al. Bacterial infection causes stress-induced memory dysfunction in mice. Gut 2011; 60: 307-317. doi:10.1136/gut.2009.202515

[7] Bagga D, Reichert JL, Koschutnig K, et al. Probiotics drive gut microbiome triggering emotional brain signatures. Gut Microbes 2018; 9: 486-96. doi:10.1080/19490976.2018.1 460015

[8] MacFabe DF, Cain NE, Boon F, et al. Effects of the enteric bacterial metabolic product propionic acid on object-directed behavior, social behavior, cognition, and neuroinflammation in adolescent rats: Relevance to autism spectrum disorder. Behavioural Brain Research 2011; 217: 47-54. doi:10.1016/j. bbr.2010.10.005

[9] Cheung SG, Goldenthal AR, Uhlemann AC, et al. Systematic Review of Gut Microbiota and Major Depression. Front Psychiatry 2019; 10: 34. doi:10.3389/fpsyt.2019.00034

[10] Valles-Colomer M, Falony G, Darzi Y, et al. The neuroactive potential of the human gut microbiota in quality of life and depression. Nat Microbiol 2019; 4: 623-32. doi:10.1038/ s41564-018-0337-x

[11] Breen DP, Halliday GM, Lang AE. Gut-brain axis and the spread of alpha-synuclein pathology: Vagal highway or dead end? Mov Disord 2019; 34: 307-16. doi:10.1002/mds.27556 
[12] Lee YK, Menezes JS, Umesaki Y, et al. Proinflammatory T-cell responses to gut microbiota promote experimental autoimmune encephalomyelitis. Proceedings of the National Academy of Sciences of the United States of America 2011; 108 Suppl 1: 4615-22. doi:10.1073/pnas.1000082107

[13] Erny D, Hrabe de Angelis, AL, Jaitin D, et al. Host microbiota constantly control maturation and function of microglia in the CNS. Nat Neurosci 2015; 18: 965-77. doi:10.1038/ nn. 4030

[14] Baj A, Moro E, Bistoletti M, et al. Glutamatergic Signaling Along The Microbiota-Gut-Brain Axis. Int J Mol Sci 2019. doi:10.3390/ijms20061482

[15] Chen GY. The Role of the Gut Microbiome in Colorectal Cancer. Clin Colon Rectal Surg 2018; 31: 192-8. doi:10.1055/s-0037-1602239

[16] Castellarin M, Warren RL, Freeman JD, et al. Fusobacterium nucleatum infection is prevalent in human colorectal carcinoma. Genome Research 2012; 22: 299-306. doi:10.1101/ gr.126516.111

[17] Kostic AD, Chun E, Robertson L, et al. Fusobacterium nucleatum potentiates intestinal tumorigenesis and modulates the tumor-immune microenvironment. Cell Host Microbe 2013; 14: 207-15. doi:10.1016/j.chom.2013.07.007

[18] Yang L, Lu X, Nossa CW, et al. Inflammation and intestinal metaplasia of the distal esophagus are associated with alterations in the microbiome. Gastroenterology 2009; 137 : 588-97. doi:10.1053/j.gastro.2009.04.046

[19] Dapito DH, Mencin A, Gwak G-Y, et al. Promotion of hepatocellular carcinoma by the intestinal microbiota and TLR4. Cancer cell 2012; 21: 504-16. doi:10.1016/j. ccr.2012.02.007

[20] Fernández MF, Reina-Pérez I, Astorga JM, et al. Breast Cancer and Its Relationship with the Microbiota. Int J Environ Res Public Health 2018. doi:10.3390/ijerph15081747

[21] Schwabe RF, Jobin C. The microbiome and cancer. Nature reviews. Cancer 2013; 13: 800-12. doi:10.1038/nrc3610

[22] Yoshimoto S, Loo TM, Atarashi K, et al. Obesity-induced gut microbial metabolite promotes liver cancer through senescence secretome. Nature 2013; 499: 97-101. doi:10.1038/ nature12347

[23] Wu S, Morin PJ, Maouyo D, et al. Bacteroides fragilis enterotoxin induces C-Myc expression and cellular proliferation. Gastroenterology 2003; 124: 392-400. doi:10.1053/ gast.2003.50047

[24] Grivennikov S, Karin E, Terzic J, et al. IL-6 and Stat3 are required for survival of intestinal epithelial cells and development of colitis-associated cancer. Cancer cell 2009; 15: 103-13. doi:10.1016/j.ccr.2009.01.001

[25] Rubinstein MR, Wang X, Liu W, et al. Fusobacterium nucleatum promotes colorectal carcinogenesis by modulating $\mathrm{E}$-cadherin/ $\beta$-catenin signaling via its FadA adhesin. Cell Host Microbe 2013; 14: 195-206. doi:10.1016/J. CHOM.2013.07.012

[26] Pandiyan P, Bhaskaran N, Zou M, et al. Microbiome Dependent Regulation of Tregs and Th17 Cells in Mucosa. Front Immunol 2019; 10: 426. doi:10.3389/fimmu.2019.00426

[27] Faith J], Ahern PP, Ridaura VK, et al. Identifying gut microbe-host phenotype relationships using combinatorial communities in gnotobiotic mice. Science translational medicine 2014; 6: 220ra11. doi:10.1126/scitranslmed.3008051

[28] Louveau A, Smirnov I, Keyes T], et al. Structural and functional features of central nervous system lymphatic vessels. Nature 2015; 523: 337-41. doi:10.1038/nature14432
[29] Bartholomäus I, Kawakami N, Odoardi F, et al. Effector $T$ cell interactions with meningeal vascular structures in nascent autoimmune CNS lesions. Nature 2009; 462: 94-8. doi:10.1038/nature08478

[30] Hanwehr RI von, Hofman FM, Taylor CR, et al. Mononuclear lymphoid populations infiltrating the microenvironment of primary CNS tumors. Characterization of cell subsets with monoclonal antibodies. J Neurosurg 1984; 60: 1138-47. doi:10.3171/jns.1984.60.6.1138

[31] Atarashi K, Tanoue T, Shima T, et al. Induction of colonic regulatory T cells by indigenous Clostridium species. Science 2011; 331: 337-41. doi:10.1126/science.1198469

[32] Furusawa Y, Obata Y, Fukuda S, et al. Commensal microbe-derived butyrate induces the differentiation of colonic regulatory T cells. Nature 2013; 504: 446-50. doi:10.1038/ nature 12721

[33] Pandiyan P, Zheng L, Ishihara S, et al. CD4 + CD25 + Foxp3 + regulatory T cells induce cytokine deprivation-mediated apoptosis of effector CD4 + T cells. Nat Immunol 2007; 8: 1353-62. doi:10.1038/ni1536

[34] Ivanov II, Atarashi K, Manel N, et al. Induction of intestinal Th17 cells by segmented filamentous bacteria. Cell 2009; 139: 485-98. doi:10.1016/j.cell.2009.09.033

[35] Round JL, Lee SM, Li ], et al. The Toll-like receptor 2 pathway establishes colonization by a commensal of the human microbiota. Science 2011; 332: 974-7. doi:10.1126/science. 1206095

[36] Mazmanian SK, Liu CH, Tzianabos AO, et al. An immunomodulatory molecule of symbiotic bacteria directs maturation of the host immune system. Cell 2005; 122: 107-18. doi:10.1016/j.cell.2005.05.007

[37] Viaud S, Daillere R, Boneca IG, et al. Gut microbiome and anticancer immune response: really hot Sh*t! Cell Death Differ 2015; 22: 199-214. doi:10.1038/cdd.2014.56

[38] Schirmer M, Smeekens SP, Vlamakis H, et al. Linking the Human Gut Microbiome to Inflammatory Cytokine Production Capacity. Cell 2016; 167: 1125-1136.e8. doi:10.1016/j. cell.2016.10.020

[39] Chen Z, Feng X, Herting C], et al. Cellular and Molecular Identity of Tumor-Associated Macrophages in Glioblastoma. Cancer Research 2017; 77: 2266-78. doi:10.1158/00085472.CAN-16-2310

[40] Fecci PE, Mitchell DA, Whitesides JF, et al. Increased regulatory T-cell fraction amidst a diminished CD4 compartment explains cellular immune defects in patients with malignant glioma. Cancer Res 2006; 66: 3294-302. doi:10.1158/00085472.CAN-05-3773

[41] Lohr J, Ratliff T, Huppertz A, et al. Effector T-cell infiltration positively impacts survival of glioblastoma patients and is impaired by tumor-derived TGF-beta. Clin Cancer Res 2011; 17: 4296-308. doi:10.1158/1078-0432.CCR-10-2557

[42] Han S, Ma E, Wang X, et al. Rescuing defective tumor-infiltrating T-cell proliferation in glioblastoma patients. Oncology Letters 2016; 12: 2924-9. doi:10.3892/ol.2016.4944

[43] Badie B, Schartner JM. Flow cytometric characterization of tumor-associated macrophages in experimental gliomas. Neurosurgery 2000; 46: 957-61; discussion 961-2. doi:10.1097/00006123-200004000-00035

[44] Dutoit V, Herold-Mende C, Hilf N, et al. Exploiting the glioblastoma peptidome to discover novel tumour-associated antigens for immunotherapy. Brain 2012; 135: 1042-54. doi:10.1093/brain/aws042

[45] Beckhove P, Warta R, Lemke B, et al. Rapid T cell-based identification of human tumor tissue antigens by automated 
two-dimensional protein fractionation. J Clin Invest 2010; 120: 2230-42. doi:10.1172/JCI37646

[46] Albulescu R, Codrici E, Popescu ID, et al. Cytokine patterns in brain tumour progression. Mediators Inflamm 2013; 2013: 979748. doi:10.1155/2013/979748

[47] Nijaguna MB, Patil V, Hegde AS, et al. An Eighteen Serum Cytokine Signature for Discriminating Glioma from Normal Healthy Individuals. PloS one 2015; 10: e0137524. doi:10.1371/journal.pone. 0137524

[48] Asseman C, Mauze S, Leach MW, et al. An essential role for interleukin 10 in the function of regulatory $T$ cells that inhibit intestinal inflammation. J Exp Med 1999; 190: 995-1004. doi:10.1084/jem.190.7.995

[49] Nakamura K, Kitani A, Fuss I, et al. TGF-beta 1 plays an important role in the mechanism of $C D 4+C D 25+$ regulatory $T$ cell activity in both humans and mice. Journal of immunology 2004; 172: 834-42. doi:10.4049/jimmunol.172.2.834

[50] Tai X, van Laethem F, Pobezinsky L, et al. Basis of CTLA-4 function in regulatory and conventional CD4(+) T cells. Blood 2012; 119: 5155-63. doi:10.1182/blood-2011-11-388918

[51] Crane CA, Ahn B], Han SJ, et al. Soluble factors secreted by glioblastoma cell lines facilitate recruitment, survival, and expansion of regulatory T cells: implications for immunotherapy. Neuro Oncol 2012; 14: 584-95. doi:10.1093/neuonc/ nos 014

[52] Wainwright DA, Balyasnikova IV, Chang AL, et al. IDO expression in brain tumors increases the recruitment of regulatory T cells and negatively impacts survival. Clinical Cancer Research 2012; 18: 6110-21. doi:10.1158/10780432.CCR-12-2130

[53] Heimberger AB, Abou-Ghazal M, Reina-Ortiz C, et al. Incidence and prognostic impact of FoxP3 + regulatory $\mathrm{T}$ cells in human gliomas. Clin Cancer Res 2008; 14: 5166-72. doi:10.1158/1078-0432.CCR-08-0320

[54] Zou JP, Morford LA, Chougnet C, Dix AR, Brooks AG, Torres $\mathrm{N}$, et al. Human glioma-induced immunosuppression involves soluble factor(s) that alters monocyte cytokine profile and surface markers. J Immunol. 1999;162:4882-92.

[55] Seliger C, Leukel P, Moeckel S, et al. Lactate-modulated induction of THBS-1 activates transforming growth factor (TGF)-beta2 and migration of glioma cells in vitro. PloS one 2013; 8: e78935. doi:10.1371/journal.pone.0078935

[56] Silginer M, Burghardt I, Gramatzki D, et al. The aryl hydrocarbon receptor links integrin signaling to the TGF-beta pathway. Oncogene 2016; 35: 3260-71. doi:10.1038/ onc. 2015.387

[57] Tanabe K, Matsushima-Nishiwaki R, Yamaguchi S, et al. Mechanisms of tumor necrosis factor-alpha-induced interleukin-6 synthesis in glioma cells. J Neuroinflammation. 2010; 7: 16. doi:10.1186/1742-2094-7-16

[58] Zheng X, Zhou K, Zhang Y, et al. Food withdrawal alters the gut microbiota and metabolome in mice. FASEB J 2018; 32: 4878-88. doi:10.1096/fj.201700614R

[59] Garnier D, Renoult O, Alves-Guerra M-C, et al. Glioblastoma Stem-Like Cells, Metabolic Strategy to Kill a Challenging Target. Front Oncol 2019; 9: 118. doi:10.3389/ fonc. 2019.00118

[60] Kelly C], Zheng L, Campbell EL, et al. Crosstalk between Microbiota-Derived Short-Chain Fatty Acids and Intestinal Epithelial HIF Augments Tissue Barrier Function. Cell Host Microbe 2015; 17: 662-71. doi:10.1016/j. chom.2015.03.005

[61] Arpaia N, Campbell C, Fan X, et al. Metabolites produced by commensal bacteria promote peripheral regulatory
T-cell generation. Nature 2013; 504: 451-5. doi:10.1038/ nature12726

[62] Cervenka I, Agudelo LZ, Ruas JL. Kynurenines: Tryptophan's metabolites in exercise, inflammation, and mental health. Science 2017. doi:10.1126/science.aaf9794

[63] Lee JH, Lee J. Indole as an intercellular signal in microbial communities. FEMS Microbiol Rev 2010; 34: 426-44. doi:10.1111/j.1574-6976.2009.00204.x

[64] Yano JM, Yu K, Donaldson GP, et al. Indigenous bacteria from the gut microbiota regulate host serotonin biosynthesis. Cell 2015; 161: 264-76. doi:10.1016/j.cell.2015.02.047

[65] Clarke G, Grenham S, Scully P, et al. The microbiome-gut-brain axis during early life regulates the hippocampal serotonergic system in a sex-dependent manner. Mol Psychiatry 2013; 18: 666-73. doi:10.1038/mp.2012.77

[66] Desbonnet L, Garrett L, Clarke G, et al. The probiotic Bifidobacteria infantis: An assessment of potential antidepressant properties in the rat. Journal of Psychiatric Research 2008; 43: 164-74. doi:10.1016/j.jpsychires.2008.03.009

[67] Valladares R, Bojilova L, Potts AH, et al. Lactobacillus johnsonii inhibits indoleamine 2,3-dioxygenase and alters tryptophan metabolite levels in BioBreeding rats. FASEB J 2013; 27: 1711-20. doi:10.1096/fj.12-223339

[68] Raison CL, Dantzer R, Kelley KW, et al. CSF concentrations of brain tryptophan and kynurenines during immune stimulation with IFN-alpha: relationship to CNS immune responses and depression. Mol Psychiatry 2010; 15: 393-403. doi:10.1038/mp.2009.116

[69] Wikoff WR, Anfora AT, Liu J, et al. Metabolomics analysis reveals large effects of gut microflora on mammalian blood metabolites. Proc Natl Acad Sci U S A 2009; 106: 3698-703. doi:10.1073/pnas.0812874106

[70] Opitz CA, Litzenburger UM, Sahm F, et al. An endogenous tumour-promoting ligand of the human aryl hydrocarbon receptor. Nature 2011; 478: 197-203. doi:10.1038/nature 10491

[71] Pilotte L, Larrieu P, Stroobant V, et al. Reversal of tumoral immune resistance by inhibition of tryptophan 2,3-dioxygenase. Proc Natl Acad Sci U S A 2012; 109: 2497-502. doi:10.1073/pnas.1113873109

[72] Uyttenhove C, Pilotte L, Theate I, et al. Evidence for a tumoral immune resistance mechanism based on tryptophan degradation by indoleamine 2,3-dioxygenase. Nat Med 2003; 9: 1269-74. doi:10.1038/nm934

[73] Fallarino F, Grohmann U, You S, et al. The combined effects of tryptophan starvation and tryptophan catabolites down-regulate $T$ cell receptor zeta-chain and induce a regulatory phenotype in naive T cells. I Immunol 2006; 176 : 6752-61. doi:10.4049/jimmunol.176.11.6752

[74] Favre D, Mold J, Hunt PW, et al. Tryptophan catabolism by indoleamine 2,3-dioxygenase 1 alters the balance of TH17 to regulatory T cells in HIV disease. Sci Transl Med 2010; 2: 32ra36. doi:10.1126/scitranslmed.3000632

[75] Frumento G, Rotondo R, Tonetti M, et al. Tryptophan-derived catabolites are responsible for inhibition of $\mathrm{T}$ and natural killer cell proliferation induced by indoleamine 2,3-dioxygenase. J Exp Med 2002; 196: 459-68. doi:10.1084/ jem.20020121

[76] Fallarino F, Grohmann U, Vacca C, et al. T cell apoptosis by tryptophan catabolism. Cell Death Differ 2002; 9: 1069-77. doi:10.1038/sj.cdd. 4401073

[77] Mezrich JD, Fechner JH, Zhang X, et al. An interaction between kynurenine and the aryl hydrocarbon receptor can 
generate regulatory T cells. J Immunol 2010; 185: 3190-8. doi:10.4049/jimmunol.0903670

[78] Rothhammer V, Mascanfroni ID, Bunse L, et al. Type I interferons and microbial metabolites of tryptophan modulate astrocyte activity and central nervous system inflammation via the aryl hydrocarbon receptor. Nat Med 2016; 22: 586-97. doi:10.1038/nm.4106

[79] Quintana F], Basso AS, Iglesias AH, et al. Control of T(reg) and $T(H) 17$ cell differentiation by the aryl hydrocarbon receptor. Nature 2008; 453: 65-71. doi:10.1038/nature 06880

[80] Opitz CA, Litzenburger UM, Opitz U, et al. The indoleamine-2,3-dioxygenase (IDO) inhibitor 1-methyl-D-tryptophan upregulates IDO1 in human cancer cells. PloS one 2011; 6: e19823. doi:10.1371/journal.pone.0019823

[81] Vezzani A, Gramsbergen JB, Speciale C, et al. Production of quinolinic acid and kynurenic acid by human glioma. Adv Exp Med Biol 1991; 294: 691-5. doi:10.1007/978-1-46845952-4_95

[82] Alberati-Giani D, Ricciardi-Castagnoli P, Köhler C, et al. Regulation of the kynurenine metabolic pathway by interferon-gamma in murine cloned macrophages and microglial cells. J Neurochem 1996; 66: 996-1004. doi:10.1046/j.14714159.1996.66030996.x

[83] D’Amato NC, Rogers T], Gordon MA, et al. A TDO2-AhR signaling axis facilitates anoikis resistance and metastasis in triple-negative breast cancer. Cancer Res 2015; 75: 4651-64. doi:10.1158/0008-5472.CAN-15-2011

[84] McCord M, Mukouyama YS, Gilbert MR, et al. Targeting WNT Signaling for Multifaceted Glioblastoma Therapy. Front Cell Neurosci 2017; 11: 318. doi:10.3389/fncel.2017.00318

[85] Carro MS, Lim WK, Alvarez M], et al. The transcriptional network for mesenchymal transformation of brain tumours. Nature 2010; 463: 318-25. doi:10.1038/nature08712

[86] West AJ, Tsui V, Stylli SS, et al. The role of interleukin-6-STAT3 signalling in glioblastoma. Oncol Lett 2018; 16: 4095-104. doi:10.3892/ol.2018.9227

[87] Ferguson SD, Srinivasan VM, Heimberger AB. The role of STAT3 in tumor-mediated immune suppression. J Neurooncol 2015; 123: 385-94. doi:10.1007/s11060-015-1731-3

[88] Leidgens V, Seliger C, Jachnik B, et al. Ibuprofen and Diclofenac Restrict Migration and Proliferation of Human Glioma Cells by Distinct Molecular Mechanisms. PloS one 2015; 10: e0140613. doi:10.1371/journal.pone.0140613
[89] Yamini B. NF-kappaB, Mesenchymal Differentiation and Glioblastoma. Cells 2018. doi:10.3390/cells7090125

[90] Gray GK, McFarland BC, Nozell SE, et al. NF-kappaB and STAT3 in glioblastoma: therapeutic targets coming of age. Expert Rev Neurother 2014; 14: 1293-306. doi:10.1586/147 37175.2014.964211

[91] Monsalve FA, Pyarasani RD, Delgado-Lopez F, et al. Peroxisome proliferator-activated receptor targets for the treatment of metabolic diseases. Mediators Inflamm 2013; 2013: 549627. doi:10.1155/2013/549627

[92] Ellis HP, Kurian KM. Biological Rationale for the Use of PPARgamma Agonists in Glioblastoma. Front Oncol 2014; 4: 52. doi:10.3389/fonc.2014.00052

[93] Grommes C, Conway DS, Alshekhlee A, et al. Inverse association of PPARgamma agonists use and high grade glioma development. J Neurooncol 2010; 100: 233-9. doi:10.1007| s11060-010-0185-x

[94] Maleki Vareki S, Rytelewski M, Figueredo R, et al. Indoleamine 2,3-dioxygenase mediates immune-independent human tumor cell resistance to olaparib, gamma radiation, and cisplatin. Oncotarget 2014; 5: 2778-91. doi:10.18632/ oncotarget.1916

[95] Fulda S, Kogel D. Cell death by autophagy: emerging molecular mechanisms and implications for cancer therapy. Oncogene 2015; 34: 5105-13. doi:10.1038/onc.2014.458

[96] Joseph JV, Conroy S, Tomar T, et al. TGF- $\beta$ is an inducer of ZEB1-dependent mesenchymal transdifferentiation in glioblastoma that is associated with tumor invasion. Cell Death \& Disease 2014; 5: e1443. doi:10.1038/cddis.2014.395

[97] Bhat KPL, Balasubramaniyan V, Vaillant B, et al. Mesenchymal differentiation mediated by NF-kB promotes radiation resistance in glioblastoma. Cancer cell 2013; 24: 331-346 doi:10.1016/j.ccr.2013.08.001

[98] Rutledge WC, Kong J, Gao J, et al. Tumor-Infiltrating Lymphocytes in Glioblastoma Are Associated with Specific Genomic Alterations and Related to Transcriptional Class. Clinical Cancer Research 2013; 19: 4951-4960. doi:10.1158/10780432.CCR-13-0551

Bibliografie

DOI https://doi.org/10.1055/a-1037-2271

Nervenheilkunde 2020; 39: 31-39

(c) Georg Thieme Verlag KG Stuttgart · New York ISSN 0722-1541 
Punkte sammeln auf CME.thiemede

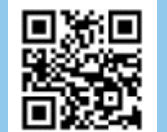

Diese Fortbildungseinheit ist in der Regel 12 Monate online für die Teilnahme verfügbar. Den genauen Einsendeschluss finden Sie unter https://eref.thieme.de/CXE1XKW.

Sollten Sie Fragen zur Online-Teilnahme haben, finden Sie unter https://cme.thieme.de/hilfe eine ausführliche Anleitung. Wir wünschen viel Erfolg beim Beantworten der Fragen!

Unter https://eref.thieme.de/CXE1XKW oder über den QR-Code kommen Sie direkt zur Startseite des Wissenstests.

VNR 2760512020158722252 trbildu

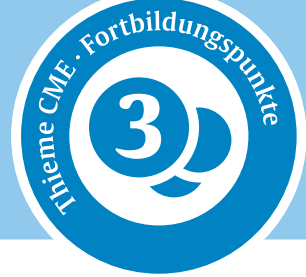

\section{Frage 1}

Welche Aussage zum Glioblastom ist richtig?

A Der Anteil von Glioblastomen an allen hirneigenen Hirntumoren ist eher gering.

B Die meisten Glioblastome entwickeln sich sekundär aus initial benignen Gliomen.

C Trotz aktueller Standardtherapie liegt das mediane Überleben beim Glioblastom in Studien bei etwa 20,9 Monaten.

D Es handelt sich um insgesamt sehr seltene Tumoren (Inzidenz $<1 / 1000000)$.

E Auch in fortgeschrittenen Stadien sind die betroffenen Patienten klinisch kaum auffällig.

\section{Frage 2}

Was verhindert eine direkte Interaktion zwischen Mikrobiota und ZNS?

A Das vollständige Fehlen von Bestandteilen des Immunsystems im ZNS.

B Schranken beim Übergang zwischen Darm und Blut sowie zwischen Blut und ZNS.

C Kurze Lebensdauer von Bakterien im Darm.

D Bakterielle Signale können von Astrozyten nicht verarbeitet werden.

E Die Besiedlung des Körpers durch Mikrobiota stellt eine absolute Ausnahme dar.

\section{Frage 3}

Was versteht man unter bakterieller Translokation?

A Expression bakterieller Bestandteile an der Außenseite der Zellwand.

B Produktion von Botenstoffen, die eine Entzündungsreaktion hemmen.

C Migration von Bakterien oder bakteriellen Bestandteilen vom Darmlumen zum Epithel, mesenterischen Lymphknoten oder anderen extraintestinalen Organen.

D Eintritt von Bakterien in das Blut über unphysiologische Eintrittsstellen (z. B. offene Wunden).

E Aktivierung spezifischer T-Zellen durch Bakterien.

\section{Frage 4}

Wobei handelt es sich um keinen gängigen Erklärungsansatz, wie Darmmikrobiota zu Veränderungen im ZNS führen können?

A Aktive Migration ins Hirnparenchym zur Aktivierung von Signalwegen.

B Aktivierung des Nervus vagus.

C Neuroimmunologische Signalwege.

D Mikrobielle Metaboliten.

E Veränderungen im glutaminergen System.

\section{Frage 5}

Welche Aussage zur Interaktion zwischen Mikrobiota und Immunsystem ist falsch?

A Das mikrobielle Wachstum im Darm wird unter anderem durch mukosaassoziierte Th17-Zellen sowie durch die Produktion von IgA kontrolliert.

B Mikrobiota können die Entwicklung und Funktion lokaler Immunzellen wie Th17- und regulatorischen T-Zellen regulieren.

C Durch Mikrobiota induzierte T-Zellen können auch an extraintestinalen Organen zu Wirkungen führen.

D Die Zytokinkonzentrationen im Blut sind abhängig von der mikrobiellen Zusammensetzung im Darm.

E Interaktionen zwischen Mikrobiota und Immunsystem beschränken sich ausschließlich auf die Darmmukosa.

\section{Frage 6}

Welcher der folgenden Zelltypen ist kein bekannter Teil des Immunzellinfiltrats beim Glioblastom?
A Regulatorische T-Zellen
B CD4-positive Effektor-T-Zellen
C Tumorassoziierte Makrophagen
D Becherzellen
E CD8-positive Zellen 


\section{Punkte sammeln auf CM/E.thieme.de}

Fortsetzung $\ldots$

\section{Frage 7}

Welches der folgenden Stoffwechselprodukte spielt nach aktuellem Stand wahrscheinlich keine Rolle bei einer möglichen Interaktion zwischen Darmmikrobiota und Hirntumoren?
A Kynurenin
B Kurzkettige Fettsäuren
C Indole
D Noradrenalin
E Tryptophan

\section{Frage 8}

Welche Aussage zu Indolamin-2,3-Dioxygenase (IDO) und Tryptophan-2,3-Dioxygenase (TDO) ist richtig?

A Es handelt sich um Enzyme des Tryptophanstoffwechsels, die bei Hirntumoren möglicherweise eine Funktion bei der Aufrechterhaltung des immunsuppressiven Milieus übernehmen.

B Es handelt sich um Enzyme, die für eine adäquate Immunantwort unverzichtbar sind.

C Beide Enzyme werden insbesondere im Darmepithel exprimiert.

D Durch Hemmung bestimmter Signalwege sind TDO und IDO Teil eines wichtigen Abwehrmechanismus zur Tumorbekämpfung.

E Es handelt sich um bakterielle Enzyme, die für den Abbau von Serotonin wichtig sind.

\section{Frage 9}

Welche Aussage zu Signalwegen beim Glioblastom ist richtig?

A Anders als bei anderen soliden Tumoren konnten beim Glioblastom kaum relevante Signalwege identifiziert werden.

B Die geringe Proliferationsrate bei Glioblastomen ist entscheidend für die Resistenz gegen Strahlentherapie.

C Beim STAT3 bzw. dem JAK/STAT-Signalweg handelt es sich um einen Knotenpunkt der Tumorentwicklung beim Glioblastom, der unter anderem durch Zytokine wie Interleukin 6 induziert werden kann.

D Es konnten einige Signalwege identifiziert werden, die durch bakterielle Bestandteile aktiviert werden können.

E NF-kB spielt bei Hirntumoren praktisch keine Rolle.

\section{Frage 10}

Welche Aussage zum mesenchymalen Phänotyp beim Glioblastom ist falsch?

A Der mesenchymale Phänotyp zeichnet sich durch eine erhöhte Invasivität der Tumorzellen aus.

B Das Vorliegen eines mesenchymalen Phänotyps ist mit einer schlechteren Prognose assoziiert.

C Der mesenchymale Phänotyp ist insbesondere durch eine stark herabgesetzte Proliferation der Tumorzellen gekennzeichnet.

D Die Aktivierung bestimmter Signalwege wie beispielsweise STAT3-abhängiger Mechanismen kann zu einer Transdifferenzierung von einem proneuralen hin zu einem mesenchymalen Phänotyp führen.

E Der $\beta$-Catenin/Wnt-Signalweg spielt eine Rolle bei der Entwicklung des mesenchymalen Phänotyps. 